\title{
Modelos de crecimiento diamétrico para Nothofagus obliqua
}

Diameter growth models for Nothofagus obliqua

LUIS CHAUCHARD, RENATO SBRANCIA

Universidad Nacional del Comahue, Administración de Parques Nacionales, Instituto Nacional de Tecnología Agropecuaria, Av. San Martín 188 (8370),

San Martín de los Andes, Neuquén, Argentina.

E-mail: chauchard@smandes.com.ar / orforestal@hotmail.com

\section{SUMMARY}

Non-linear, Bertalanffy-Richards-type models of diameter growth were developed for Nothofagus obliqua and fitted for different social tree strata using the diameter annual increment (id) and diameter at breast height (dap). The biological and statistical analyses were conducted from two different sources; an independent data set taken mainly from permanent sample plots and a real growth series obtained from tree cores and stem analyses. Models for different purposes were obtained through both data sets. For the prediction of short-term diameter increments, a model was obtained that related independent data of trees belonging to the same social stratum. Models of higher biological meaning were obtained by relating the growth series and their significance and are discussed here. By means of these biological models, the growth dynamics of $N$. obliqua were analyzed under different competitive status.

Key words: growth dynamics, Bertalanffy-Richards, diameter model, Nothofagus.

\section{RESUMEN}

Se han desarrollado varios modelos de crecimiento diamétrico para la especie Nothofagus obliqua. Este trabajo continúa a otros similares realizados con N. nervosa (Chauchard 1991) y N. dombeyi (Chauchard et al. 2001). El modelo seleccionado para realizar el estudio es uno no lineal usualmente denominado Bertalanffy-Richards. El mismo se ajustó a partir de tres fuentes de datos: parcelas permanentes (con sólo dos mediciones cada una), análisis fustales y tarugos de incremento. Estos datos fueron segregados por estrato social y luego para cada uno se ajustó el citado modelo con las variables incremento diamétrico (id)-diámetro a la altura del pecho (dap). Los ajustes y análisis se realizaron a partir de dos agrupamientos de los datos. Por un lado se utilizaron datos independientes que provenían principalmente de las parcelas de muestreo, mientras que por otro se emplearon series reales de crecimiento obtenidas de los tarugos y los análisis fustales. Con ambos grupos se obtienen modelos con aplicaciones diferentes. Con los datos independientes se obtienen modelos de interés para la predicción del incremento diamétrico en el corto plazo, mientras que con las series de crecimiento se obtienen modelos de una alta significancia biológica. A través de estos modelos biológicos se interpretó la dinámica de crecimiento de $N$. obliqua bajo diferentes estados de competencia.

Palabras claves: dinámica del crecimiento, Bertalanffy-Richards, modelo diamétrico, Nothofagus. 


\section{INTRODUCCION}

En 1991 se iniciaron, a partir en su mayoría de datos existentes, una serie de estudios sobre el modelado del crecimiento de las especies que componen el bosque mixto de Nothofagus del norte de la Patagonia, en Argentina. Dichos datos estaban disponibles en diversos inventarios realizados en los bosques de la región.

Se comenzó modelando el crecimiento de $N$. nervosa (Chauchard 1991), se continuó con $N$. dombeyi (Chauchard et al. 2001) y en el presente trabajo se aborda la dinámica del crecimiento de N. obliqua.

El estudio realizado se ha basado en una serie de modelos que expresan el crecimiento individual en diámetro, es decir, a nivel de árbol. Son del tipo de los modelos teóricos o biológicos, que tienen como característica importante el hecho de que están asociados con una hipótesis sobre la causa o función del fenómeno descrito por la variable de respuesta (Vanclay 1994).

Sin perder de vista el rumbo trazado, que es el de obtener, a través de la modelización, las herramientas para establecer el efecto en el crecimiento y la producción de las diferentes alternativas silviculturales factibles de aplicar a los bosques de Nothofagus, se ha intentado aprovechar la utilidad de los modelos teóricos para describir las características biológicas de las especies estudiadas, asociadas al fenómeno del crecimiento (Chauchard et al. 2001).

El estudio persigue el objetivo específico de ajustar una familia de funciones compatibles de crecimiento y rendimiento diamétrico y a través de ella interpretar aspectos de la dinámica de la competencia de la especie N. obliqua (Mirb.) Oerst., roble pellín.

Se ha optado por modelar el crecimiento diamétrico por las siguientes razones (Chauchard et al. 2001):

- Está directa y fuertemente relacionado con el tamaño del árbol: área basal, volumen y tamaño de la copa.

- Es una variable de fácil y precisa medición.

- Es la variable que está siendo empleada en el desarrollo de los diagramas de manejo de la densidad para bosques mixtos de Nothofagus de Argentina (Chauchard et al. 1994, Chauchard et al. 1999, Sbrancia et al. 1998).

\section{MATERIALES Y METODOS}

Area de estudio. El estudio abarca tres zonas que integran unidades de ordenación forestal dentro de la Reserva Nacional del Parque Nacional Lanín, en la provincia del Neuquén, Argentina. Todos los bosques en estudio se localizan en la Cuenca de los Lagos Lácar-Nonthué y abarcan una superficie de aproximadamente 1.500 ha.

Los rodales estudiados pertenecen a los tipos forestales puros y mixtos de Nothofagus nervosa (Phil.) Dim. et Mil. (raulí), N. obliqua (Mirb.) Oerst. (roble pellín) y $N$. dombeyi (Mirb.) Oerst. (coihue), creciendo en un rango altitudinal de 600 a 1.000 ms.n.m.

Fuente y análisis de los datos. Se trabajó con tres fuentes de datos:

a) Datos obtenidos en Parcelas Permanentes (PP): estas parcelas fueron medidas al inicio y al final de un período que varió entre los 4 y 9 años. El seguimiento en las parcelas se realizó a nivel de cada árbol; a cada uno se le midió el diámetro a la altura del pecho (dap) y se determinó la especie y la posición sociológica, de acuerdo a la clasificación tradicional para especies heliófilas (Spurr y Barnes 1980, Donoso 1993) adaptada por Chauchard et al. (2001).

b) Datos obtenidos en tarugos o virutas de incremento (TI): cada muestra consiste en un tarugo obtenido barrenando el árbol con el taladro de Pressler a la altura del pecho. De cada árbol barrenado se registraron los siguientes datos: especie, dap y el estrato social según los criterios antes mencionados.

c) Datos obtenidos en rodelas de árboles apeados (RO): cada muestra consiste en una torta o rodela cortada en el árbol a la altura de 1,30 m desde el suelo (altura del pecho). A cada árbol apeado se le registra la especie, el dap y el estrato social.

Determinación de los crecimientos. La información básica empleada para el desarrollo de los modelos consiste en los siguientes pares de datos: incremento del dap (id) en relación al dap y a la edad (t), y el dap en función de la edad. La edad considerada es la que posee el árbol a la altura del pecho, variable empleada para evitar las incidencias que poseen una serie de factores que actúan 
en edades tempranas del árbol; además los crecimientos en diámetro, como se ha expuesto, se miden por convención a la altura de $1,30 \mathrm{~m}$, lo que significa que cuando el dap $=0$, el árbol ya tiene una edad determinada (Chauchard et al. 2001).

La base de datos se subdividió en clases diamétricas, por un lado, para amplitudes de $5 \mathrm{~cm}$ y por otro para amplitudes de $10 \mathrm{~cm}$. Para el estudio del crecimiento se utilizaron los incrementos diametrales promedios de cada clase, datos con los cuales se realizaron los ajustes de los modelos. Los ajustes fueron ensayados tanto para los datos agrupados en clases de $5 \mathrm{~cm}$ como de $10 \mathrm{~cm}$. Además, para el desarrollo y análisis de los modelos la información se segregó en dos grupos:

- Series de crecimientos: provenientes de las mediciones de los tarugos y las rodelas. Estas se diferenciaron en: completas, cuando se tenía localizada la médula y era posible determinar las edades; e incompletas, cuando no se localizó la médula, por lo cual no era posible relacionar los crecimientos con las edades.

- Datos independientes: todos aquellos que poseían independencia entre sí. Todas las muestras provenientes de PP, TI o RO aportaron datos independientes que consistieron en la medición del último período de cada una de dichas muestras.

Los análisis se realizaron por estratos sociales y para los dos grupos de datos, precedentemente descritos. Se analizó la posibilidad de juntar los datos de los estratos dominante y codominante, siguiendo lo propuesto por Chauchard et al. (2001). De acuerdo a estudios realizados por Chauchard et al. (1996), estos estratos dominantes representan aproximadamente el $90 \%$ de la cobertura y crecimiento de los rodales naturales estudiados. Un aspecto para destacar es que los datos provienen de sitios sin intervención relativamente homogéneos, apreciación subjetiva, habida cuenta de que todavía no se han desarrollado índices cuantitativos para expresar la calidad de sitio de la especie.

Los modelos de crecimiento en diámetro fueron desarrollados independientemente para cada grupo de datos, precedentemente citados. En primer lugar, para cada grupo y estrato social se analizaron gráficamente los comportamientos de los pares de datos id-dap. En particular, a las series de crecimiento se incorporaron en un mismo gráfico todas las series correspondiente a cada estrato social, asignándole a la serie proveniente de cada árbol el estrato que poseía al momento de tomar la muestra. Con estos gráficos se obtuvo la primera impresión respecto a la posibilidad de juntar en un único estrato superior a los estratos dominante y codominante.

Realizados los análisis gráficos se pasó al ajuste de la función para los distintos grupos de datos y estratos sociales. Empleando como base los incrementos diamétricos, en función de su diámetro, se ajustó un modelo de crecimiento entre ambas variables, utilizando técnicas de regresión no lineal y lineal. A partir de dicho modelo se obtuvieron matemáticamente otros modelos de rendimiento y crecimiento en diámetro en función del tiempo. En particular, a las series de crecimientos, además, se probó el procedimiento de ajustar un modelo de rendimiento y luego derivarlo en el de crecimiento correspondiente. De estas pruebas de ajustes se fueron definiendo los mejores modelos para cada caso en función de los evaluadores estadísticos y el comportamiento biológico.

Modelo de crecimiento y rendimiento empleado. Para expresar el crecimiento diamétrico de $N$. obliqua se ha elegido la función no lineal usualmente conocida como de Bertalanffy-Richards. La elección de dicha función para el estudio del crecimiento de los bosques naturales de Nothofagus se basó en que ha demostrado ser muy flexible (Richards 1959, Osumi 1983, Zeide 1989, Vanclay 1994, Chauchard et al. 2001) para adaptarse a diversos fenómenos, especialmente al crecimiento sigmoide de árboles y rodales. Pertenece a las llamadas biológicas, y su expresión diferencial general, también conocida como del tipo Bernoulli, es:

$$
\frac{d y}{d t}=a y^{m}-b y
$$

donde:

$d y / d t=$ crecimiento instantáneo.

$a, b=$ constantes de anabolismo y catabolismo, respectivamente.

$y=$ tamaño del organismo o población (en el presente estudio es el dap).

$m \quad=$ constante de alometría. Para $0<m<1 \Rightarrow$ a, $\mathrm{b}>0$; para $m>1 \Rightarrow \mathrm{a}, \mathrm{b}<0$. 
La solución matemática de la ecuación [1] permite transformarla en la función de rendimiento respectiva, la cual, derivada, permite obtener la correspondiente ecuación de crecimiento en función del tiempo.

La función de rendimiento en dap, obtenida entonces de una función del tipo [1], es de la forma siguiente:

$$
d a p=D[1-\exp (-k t)]^{1 / 1-m}
$$

donde:

dap = diámetro a la altura del pecho.

$D$ = parámetro que representa la asíntota de la función (dap asintótico)

$t \quad=$ edad a la altura del pecho.

$k, m=$ parámetros de la ecuación. En este caso $0<m<1$.

La función de rendimiento definida por [2] es sigmoidal con una asíntota superior (D) y una inflexión dependiente del parámetro $m$.

La forma derivada de la función [2] es la siguiente:

$$
\frac{d d}{d t}=\frac{k}{1-m} D[1-\exp (-k t)]^{(1 / 1-m)-1} \exp (-k t)
$$

donde: $d d / d t=i d$, crecimiento instantáneo en dap (crecimiento corriente anual en $\mathrm{cm}$ ) a la edad $t$.

Emplear la función diferencial [1] es importante porque no se requiere de la variable edad para iniciar el estudio, pero permite finalmente obtener la evolución del crecimiento en función del tiempo. Cuando se trabaja con bosques naturales, en los cuales la determinación de la edad es una tarea dificultosa y costosa, estas funciones se transforman en una llave para comenzar la investigación de la dinámica del individuo o de la población.

El procedimiento para el desarrollo de estas funciones se realizó de esta manera: se ajustó la función [1] a los pares de datos id-dap, luego se resolvió matemáticamente, para obtener la función de rendimiento [2] y asegurar la compatibilidad entre ellas. Ello implica emplear ciertas relaciones matemáticas entre los parámetros de las funciones [1] y [2]. Una vez resuelta la ecuación [2] se derivó en la correspondiente función de crecimiento instantáneo [3].

Siguiendo a Vázquez (1988) y Chauchard et al. (2001), la función [1] se puede escribir como:

$$
i d=a\left(\operatorname{dap}^{m}-\operatorname{dap} D^{m-1}\right)
$$

Donde la variable $y$ de la ecuación [1] ha sido reemplazada por el diámetro a la altura del pecho (dap).

La función [4] es la que se utilizó para realizar los ajustes estadísticos dado que hace explícita la relación entre el crecimiento y la asíntota de la función (D) (Vázquez 1988, Chauchard 1991, Chauchard et al. 2001).

Como se expresó anteriormente, en el caso de las series de crecimientos se probaron ajustes de la función de rendimiento [2], la que luego fue derivada para obtener las funciones de crecimientos [3] y [4].

Relación entre los parámetros. A través de las relaciones matemáticas entre los parámetros de la función [2] es posible describir las características del fenómeno de crecimiento. Las relaciones empleadas, denominadas parámetros de vida, son las siguientes:

$\frac{D k}{2 m+2}$

$\frac{2 m+2}{k}$

$D m^{1 / 1-m}$

$\frac{k}{m}$

$\frac{1}{k} \ln (1-m)$

TPI: tiempo o momento en que ocurre el punto máximo (de inflexión) del crecimiento absoluto y por ende de los parámetros: DPI, MCA y CRPI. 
Evaluación estadística de los modelos ajustados. El modelo [4] es intrínsecamente no lineal a causa del parámetro $m$ que no permite la linealización, además es una función condicionada.

Para aprovechar el mayor desarrollo de los estadísticos de la regresión lineal, el procedimiento de ajuste de la función [4] se realizó en dos etapas. Este procedimiento fue propuesto por Uribe (1987) y aplicado por Vázquez (1988), Chauchard (1991) y Chauchard et al. (2001); como primera etapa se deben estimar los tres parámetros de la función [4] por regresión no lineal, luego, como segunda etapa, se vuelve a correr la función con la técnica de regresión lineal, considerando ahora como constante el parámetro $m$, ya estimado anteriormente. De esta manera se ajustó el modelo no lineal, pudiendo emplear los estadísticos de la regresión lineal para evaluarlo.

Para evaluar la bondad del ajuste no lineal se utilizaron los siguientes estadísticos: las Sumas de Cuadrados del Residuo (SCR); Error Estándar de la Estimación (EEE); Error Absoluto Medio (EAM); mientras que para la regresión lineal además se utilizó el Coeficiente de Determinación $\left(\mathrm{R}^{2}\right)$. Además, se analizó el grado de adecuación gráfica de la curva y los datos observados.

Finalmente, el ajuste era aceptado si además de cumplir con los evaluadores estadísticos antes mencionados, la significancia biológica de los parámetros obtenidos era aceptable para describir la dinámica de la especie. El hecho de tener el mejor ajuste estadístico no fue una condición suficiente para la aceptación del mismo. El programa estadístico utilizado fue el Statgraphics Plus v. 3.1.

Para la asignación de los valores de partida a los parámetros para la regresión no lineal se indagaron estudios anteriores en otras especies de Nothofagus (Chauchard 1991, Peri y Martínez 1997), pero para el caso especial del diámetro máximo o asintótico (D) se estudiaron las distribuciones diamétricas de cada estrato, en las parcelas de inventarios de rodales sin intervención. En estas distribuciones se puede apreciar cuáles son los diámetros máximos que naturalmente un árbol en un determinado estrato de competencia puede alcanzar. Este estudio de las distribuciones de frecuencias naturales facilitó el proceso de ajuste a través de dos alternativas. Una de estas alternativas de ajuste se denomina método de la asíntota conocida, puesto que el valor de la asíntota, aproximada con las frecuencias diamétricas naturales, entra como una constante en el ajuste del modelo [4]. El otro camino de ajuste empleado se puede denominar método de la asíntota libre, ya que es un parámetro más de ajuste en la ecuación [4] y a través de las frecuencias diamétricas se puede aproximar el valor de partida del parámetro para el ajuste no lineal (Chauchard et al. 2001).

\section{RESULTADOS}

La base de datos empleada quedó conformada de la siguiente manera:

a) Se obtuvieron incrementos individuales en 90 parcelas de $500 \mathrm{~m}^{2}$, cuatro de $900 \mathrm{~m}^{2}$ y tres de $1.000 \mathrm{~m}^{2}$.

b) Tarugos de viruta: se barrenaron 42 árboles.

c) Cortes de árboles apeados: se obtuvieron ocho tortas o rodelas a la altura del pecho.

Para los ajustes finalmente se dispuso de 161 datos independientes y 236 pares de datos conformados en series de crecimientos (cuadro 1).

Los datos provienen de bosques puros y mixtos de Nothofagus, cuyas alturas dominantes oscilan entre los 25 y 35 metros. Ello permite inferir una relativa homogeneidad de los sitios, basados en el supuesto de que dichas alturas expresan la potencialidad productiva. Por otro lado, al desarrollarse un modelo de crecimiento independiente de la densidad, para esta etapa del estudio, no se ha considerado la influencia de la misma en cada punto de muestreo.

Analizando los gráficos de dispersión de los pares id-dap se observó que las tendencias de los estratos dominante y codominante eran muy cercanas, y en algunos puntos se cruzaban, por lo que se decidió trabajarlos como estrato único.

Similar procedimiento se realizó en un estudio con $N$. dombeyi (Chauchard et al. 2001). Una de las razones para que las tendencias de los valores de incremento diamétrico de los estratos superiores no se diferencien podría deberse a los efectos de la subjetividad al momento de realizar la clasificación, dado el carácter de cualitativo del sistema de clasificación.

Los rangos de diámetros máximos que $N$. obliqua puede alcanzar en la zona de estudio, inferidos a través de analizar las distribuciones diamétricas que se obtuvieron de los muestreos reali- 


\section{CUADRO 1}

Datos empleados para ajustar los modelos de crecimiento diamétrico. Los mismos son: incrementos del dap promedios por clase diamétrica y para cada estrato social. n: tamaño de la muestra; id: incremento del dap en cm/año.

Data used to fit the diameter growth models; increments of diameter at breast height by diameter classes and social strata. $\mathrm{n}$ : sample size; id: increment of diameter at breast height.

\begin{tabular}{|c|r|r|r|r|r|r|r|r|r|r|r|r|}
\hline & \multicolumn{9}{|c|}{ Datos Independientes } & \multicolumn{7}{c|}{ Series de Crecimiento } \\
\cline { 2 - 13 } $\begin{array}{c}\text { Clase } \\
\text { de Dap }\end{array}$ & \multicolumn{2}{|c|}{ Superior } & \multicolumn{2}{|c|}{ Intermedio } & \multicolumn{2}{|c|}{ Suprimido } & \multicolumn{2}{|c|}{ Suprimido } & \multicolumn{2}{|c|}{ Intermedio } & \multicolumn{2}{|c|}{ Suprimido } \\
\cline { 2 - 13 } & $n$ & $i d$ & $n$ & $i d$ & $n$ & $i d$ & $n$ & $i d$ & $n$ & $i d$ & $n$ & $i d$ \\
\hline \multirow{2}{*}{2,5} & - & - & - & - & - & - & 12 & 0,45 & 9 & 0,43 & 7 & 0,39 \\
7,5 & 4 & 0,77 & 2 & 0,41 & - & - & 18 & 0,57 & 9 & 0,40 & 8 & 0,39 \\
12,5 & 6 & 0,63 & 2 & 0,26 & 3 & 0,17 & 17 & 0,54 & 9 & 0,32 & 10 & 0,22 \\
17,5 & 3 & 0,67 & 3 & 0,24 & 5 & 0,12 & 14 & 0,51 & 10 & 0,31 & 10 & 0,18 \\
22,5 & 3 & 0,66 & 2 & 0,22 & 7 & 0,12 & 13 & 0,51 & 9 & 0,31 & 7 & 0,14 \\
27,5 & 8 & 0,37 & 5 & 0,23 & - & - & 13 & 0,42 & 9 & 0,24 & - & - \\
32,5 & 9 & 0,46 & 6 & 0,17 & - & - & 14 & 0,41 & 4 & 0,28 & - & - \\
37,5 & 12 & 0,37 & 6 & 0,16 & - & - & 13 & 0,35 & 1 & 0,24 & - & - \\
42,5 & 7 & 0,26 & 2 & 0,07 & - & - & 7 & 0,26 & - & - & - & - \\
47,5 & 13 & 0,41 & - & - & - & - & 7 & 0,20 & - & - & - & - \\
52,5 & 10 & 0,24 & 3 & 0,18 & - & - & 4 & 0,20 & - & - & - & - \\
57,5 & 11 & 0,30 & - & - & - & - & 2 & 0,28 & - & - & - & - \\
62,5 & 13 & 0,21 & - & - & - & - & & & - & - & - & - \\
67,5 & 2 & 0,33 & - & - & - & - & & & - & - & - & - \\
72,5 & 14 & 0,12 & - & - & - & - & & & - & - & - & - \\
\hline
\end{tabular}

zados en dicha zona, son los siguientes: estrato superior: $70-80 \mathrm{~cm}$, estrato intermedio: $55-65 \mathrm{~cm}$ y estrato suprimido: $30-35 \mathrm{~cm}$. Ello permitió aproximar el parámetro asintótico D para los modelos de los distintos estratos sociales. Esta diferenciación en los valores del parámetro asintótico para árboles de diferentes estratos sociales se asienta en lo demostrado por Chauchard (1991) y Chauchard et al. (2001), respecto al no cumplimiento de la hipótesis de asíntota común para el estudio del crecimiento de árboles de diferentes estratos de competencia.

Los resultados de los ajustes se diferenciarán según la fuente de datos que se emplee, ya sean datos independientes o series de crecimientos.

Consideraciones sobre los modelos ajustados con los datos independientes. En el ajuste de la función [4] siempre se tuvo que emplear el método de la asíntota conocida, pues los ajustes, dejando los tres parámetros $a, D$ y $m$ libres, fueron inconsistentes, valores negativos de $m$ y extremadamente altos de $D$, por ejemplo de $300 \mathrm{~cm}$.
Bajo el primer procedimiento, entonces, se emplearon valores de diámetros asintóticos para el estrato superior de $76 \mathrm{~cm}$, para el intermedio de $57 \mathrm{~cm}$ y para el suprimido de $37,5 \mathrm{~cm}$. Estos valores se consideraron naturales a partir de los registros que se poseen de la zona de estudio.

Para describir la evolución de los crecimientos de árboles de diferentes estratos sociales se decidió emplear los parámetros $a, D$ y $m$ fijados para la regresión no lineal (cuadro 2), que no variaron significativamente de los obtenidos por regresión lineal (cuadro 3).

Los resultados fueron satisfactorios, tanto en los estadísticos como en las bondades gráficas de los ajustes (figuras 1a, 1b y 1c). Los errores estándares obtenidos se consideran muy aceptables, para todos los estratos fueron menores a 0,1 cm/año. Además, estos desvíos se obtienen de ajustes con coeficientes de determinación superiores al 90\% (cuadro 3). Las tendencias de las nubes de puntos de todos los estratos sociales resultaron claras y diferenciales entre ellas (figuras 1a, 1b, 1c). 


\section{CUADRO 2}

Parámetros y estadísticos obtenidos con el grupo de datos independientes, por regresión no lineal de la función [4] y con el parámetro m libre. EAM: Error

Absoluto Medio; EEE: Error Estándar de la

Estimación; SCR: Suma de Cuadrados Residuales.

Parameters and statistics from independent data sets from a non-linear fitting of equation [4], with parameter $\mathrm{m}$ being free. EAM: Mean Absolute Error; EEE: Standard Error of Estimation; SCR: Sum of Residual Squares.

\begin{tabular}{|l|c|c|c|}
\hline Estrato & Parámetro $a$ & Parámetro $D$ & Parámetro $m$ \\
\hline Superior & 0,595530 & 76,0 & 0,1266410 \\
Intermedio & 0,358269 & 57,0 & 0,0717625 \\
Suprimido & 0,235253 & 37,5 & 0,0416074 \\
\hline Estrato & $E A M[\mathrm{~cm}]$ & $E E E[\mathrm{~cm}]$ & $S C R\left[\mathrm{~cm}^{2}\right]$ \\
\hline Superior & 0,0738 & 0,0923 & 0,0853 \\
Intermedio & 0,0477 & 0,0645 & 0,0292 \\
Suprimido & 0,0349 & 0,0433 & 0,0244 \\
\hline
\end{tabular}

\section{CUADRO 3}

Parámetros y estadísticos obtenidos con el grupo de datos independientes, por regresión lineal múltiple de la función [4] con el parámetro m constante para cada estrato social. $\mathrm{R}^{2}$ : Coeficiente de Determinación; Prueba F: Análisis de la Varianza.

Parameters and statistics obtained from independent data sets from a multiple linear fitting of equation [4], with parameter

m as a constant for each $N$. obliqua social stratum.

$\mathrm{R}^{2}$ : Coefficient of Determination; Prueba F: Analysis of Variance.

\begin{tabular}{|l|c|c|c|}
\hline Estrato & Parámetro $a$ & Parámetro $D$ & Parámetro $m$ \\
\hline Superior & 0,595530 & 76,0 & 0,1266410 \\
Intermedio & 0,322761 & 68,0 & 0,0717625 \\
Suprimido & 0,230759 & 38,0 & 0,0416074 \\
\hline Estrato & $R^{2}[\%]$ & EEE [cm] & Prueba $F$ \\
\hline Superior & 96,6 & 0,0923 & $158 * *$ \\
Intermedio & 95,5 & 0,0549 & $86^{* *}$ \\
Suprimido & 91,0 & 0,0433 & $71^{* *}$ \\
\hline
\end{tabular}

Nota: ** resultado significativo al $99 \%$ de probabilidad.
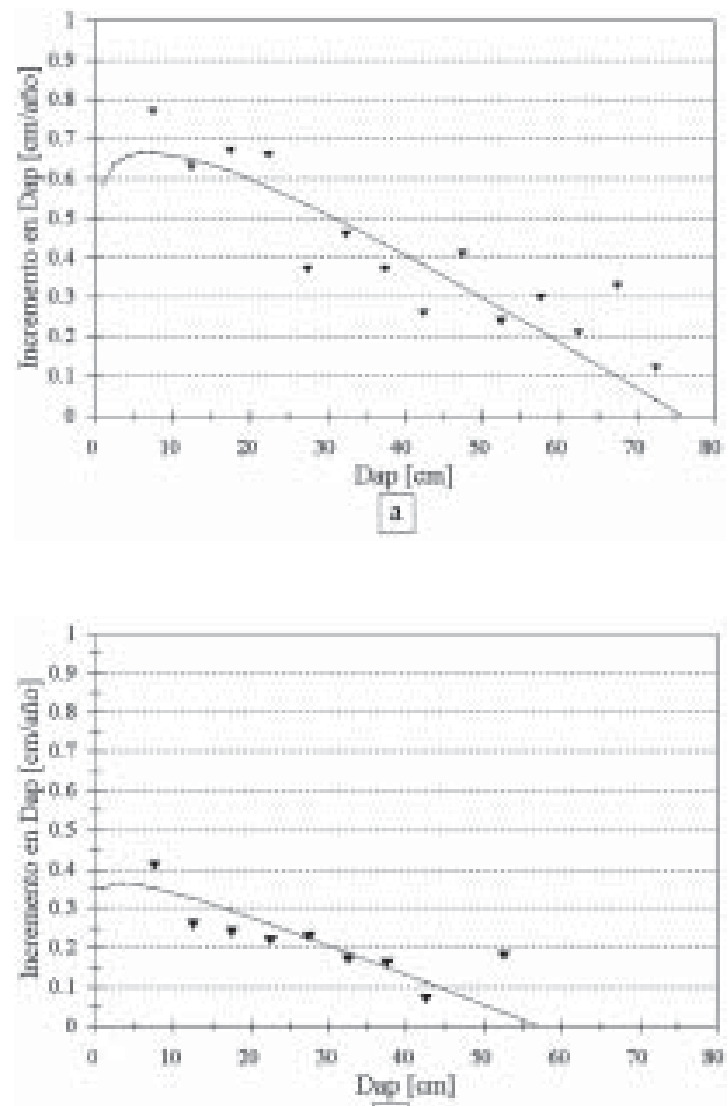

b

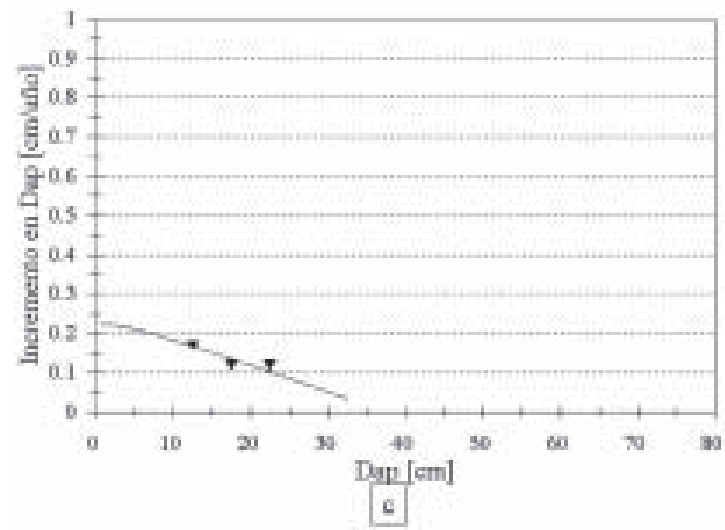

Figura 1: Función [4] ajustada a los valores de incrementos diamétricos anuales a partir del grupo de datos independientes, para diferentes estratos sociales: a) estrato superior; b) estrato intermedio y c) estrato suprimido.

Parameters and statistics obtained from a multiple linear fitting of equation [4] of the average real growth series, with parameter $\mathrm{m}$ as a constant for each $N$. obliqua social stratum. 
Estos modelos se basan en la hipótesis de que el árbol no cambia su posición social de competencia a lo largo de su vida. Bajo esta incierta hipótesis se obtuvieron resultados que determinaron que los tiempos de culminación de los incrementos corrientes anuales en diámetro (ICA) se producen a edades tempranas y sin diferenciación entre los estratos superior e intermedio, de 11 y 10 años, a la altura del pecho, respectivamente (cuadro 4).

Como se ha expresado, los modelos de crecimiento desarrollados a partir del grupo de datos independientes expresan la evolución de un árbol que, creciendo en condiciones naturales, no cambia de estrato sociológico a lo largo de su vida. Hecho éste, que se espera poco probable de ocurrir en un bosque natural, a excepción de algunos árboles dominantes $\mathrm{y}$, con menor probabilidad, suprimidos. Pero la principal utilidad de los modelos de este tipo es su utilización para la predicción del incremento corriente anual de un árbol de determinado estrato social, cualquiera sea su tamaño, más que en intentar describir su historia o la dinámica del crecimiento.

En un estudio similar realizado con $N$. dombeyi (Chauchard et al. 2001) se concluyó que para que el modelo pueda estimar los incrementos diametrales de árboles maduros, el valor de la asíntota de la función debería sobrepasar a los valores máximos de diámetros que son posibles de encontrar naturalmente. Esta conveniencia no se ha podido lograr en el presente estudio con $N$. obliqua, para el cual se han desarrollado los modelos de crecimiento, cuyos parámetros poseen todos significancia biológica, incluyendo la asíntota.

La culminaciones tan tempranas estimadas para esta especie señalan el carácter de intolerante; ello se puede apreciar especialmente con los árboles suprimidos cuyas prematuras culminaciones indican la poca capacidad de crecer bajo sombra. Un individuo suprimido de $N$. obliqua culminaría el incremento diamétrico a los 6 años a la altura del pecho, que representa un $55 \%$ de la edad de culminación de los estratos superiores (cuadros 4 y 7).

Una situación similar se determinó en un estudio anterior para los árboles suprimidos de $N$. dombeyi (Chauchard et al. 2001), donde se estimó una culminación del incremento corriente en diámetro a los 14 años a la altura del pecho, que representa un $42 \%$ de la edad de culminación del resto de los estratos. El orden de culminación del incremento en diámetro está en armonía con los requerimientos de las especies por la luz (Klepac 1983). De la misma forma que se presentó en $N$. dombeyi, los estratos dominantes e intermedios de $N$. obliqua presentaron culminaciones a edades similares (cuadros 4 y 7 ).

En virtud de los resultados obtenidos por Chauchard (1991), Chauchard et al. (2001) y en el presente estudio, se establece el temperamento más intolerante de $N$. obliqua respecto de $N$. dombeyi y, a la vez, de ambos respecto de $N$. nervosa, especie esta última cuyas culminaciones se determinaron a los 56 años para el estrato superior y a los 38 años para el estrato inferior (Chauchard 1991). También Donoso (1993) cita la mayor tolerancia de $N$. nervosa respecto de $N$. obliqua y $N$. dombeyi.

\section{CUADRO 4}

Parámetros de la función de rendimiento [2] para el grupo de datos independientes de N. obliqua y los parámetros biológicos relacionados. Máx. ICA: Máximo Incremento Corriente Anual en Diámetro.

Parameters of function [2] for the N. obliqua independent data set and related biological parameters. Max. ICA: Maximum Diameter Current Annual Increment.

\begin{tabular}{|l|c|c|c|c|c|c|c|c|c|}
\hline Estrato & $m$ & $D$ & $k$ & $\begin{array}{c}\text { MCA } \\
{[\mathrm{cm} / \mathrm{año}]}\end{array}$ & $\begin{array}{c}\text { TV } \\
{[\mathrm{años}]}\end{array}$ & $\begin{array}{c}\text { DPI } \\
{[\mathrm{cm}]}\end{array}$ & $\begin{array}{c}\text { CRPI } \\
{[\%]}\end{array}$ & $\begin{array}{c}\text { Máx.ICA } \\
{[\mathrm{cm} / \mathrm{año}]}\end{array}$ & $\begin{array}{c}\text { TPI } \\
{[\mathrm{años}]}\end{array}$ \\
\hline Superior & 0,1266410 & 76,0 & 0,01184325 & 0,40 & 190 & 7,1 & 9,3 & 0,667 & 11 \\
Intermedio & 0,0717625 & 57,0 & 0,00779829 & 0,21 & 275 & 3,3 & 10,8 & 0,353 & 10 \\
Suprimido & 0,0416074 & 37,5 & 0,00699099 & 0,13 & 298 & 1,4 & 16,8 & 0,228 & 6 \\
\hline
\end{tabular}


Este autor observó que en rodales regulares de 4050 años de edad $N$. nervosa sigue regenerando, mientras que los renovales de $N$. obliqua mueren rápidamente. No se han podido encontrar referencias comparativas entre las tolerancia de $N$. obliqua y $N$. dombeyi. Pero el mismo autor cita que el fenómeno de autorraleo es más intenso en rodales de $N$. obliqua que en los de $N$. dombeyi, que puede mantener una alta densidad de plantas hasta edades avanzadas, lo que está indicando la mayor tolerancia de esta última especie a la competencia.

Pero como se dijo, los valores anteriormente discutidos de los parámetros son para un individuo que no cambia su estrato social, ya que si lo hiciera, cosa probable en bosques naturales, se podrían dar culminaciones múltiples, dados por el ocasional aumento o descenso de los incrementos originados por la repentina liberación o paulatina opresión.

Aunque los modelos de $N$. obliqua desarrollados a partir de los datos independientes mantengan las significancias biológicas de todos sus parámetros, hay algunos aspectos del comportamiento de las funciones que presentan ciertas inconsistencias, como lo son los incrementos similares que presentan los estratos superior e intermedio, entre las edades de 140 y 190 años de vida. Se supone que en estos resultados pueden estar influyendo algunos errores al momento de clasificar las plantas entre los estratos sociales codominante e intermedio. Si se analizan los incrementos registrados entre ambos estratos entre los diámetros de 30 y $60 \mathrm{~cm}$, éstos son bastante similares. También puede estar influyendo la falta de un mayor número de muestras de árboles maduros.

Lo anterior no invalida las funciones y se espera que no sea preocupante la posible sobre o subestimación del incremento para edades cercanas a los 200 años, sobre todo considerando que a estas edades el rodal de $N$. obliqua alcanza una homogeneidad estructural a expensas de un eficiente trabajo de autorraleo en etapas tempranas, lo que ubica a casi todos sus individuos en condiciones estables de dominancia. El fenómeno de autorraleo en los bosques regulares de $N$. obliqua es tan intenso que a los 40-50 años de edad ya es posible encontrar un bosque sensiblemente regular automanejado (Donoso 1993).

Además se debe recordar que la utilidad de estos ajustes es la de proveer una predicción para el corto plazo y para un determinado momento de la vida del árbol. Ello significa que permitirá predecir para períodos de hasta 10 años el incremento diamétrico de un árbol que, con determinado diámetro, ocupa un determinado estrato social; el hecho que el árbol crezca para un siguiente período de 10 años tal como lo predice la función, dependerá de que se mantenga en su estrato social y ello ya es más difícil de pronosticar.

En definitiva, a través de estas funciones no se pretende emplear la variable edad como predictora, lo que realmente vale es que nos están indicando que un árbol de $N$. obliqua dominante de 55-60 $\mathrm{cm}$ puede estar creciendo con la misma magnitud que uno intermedio de $35 \mathrm{~cm}$.

Consideraciones sobre los Modelos Ajustados con las Series de Crecimientos. Los ajustes de la función [4] han tenido buenos estadísticos (cuadros 5 y 6) y bondades gráficas (figuras $2 \mathrm{a}, 2 \mathrm{~b}, 2 \mathrm{c}$ ). Una aceptable coincidencia hubo entre los parámetros $a$ y $D$, cuando los ajustes se realizaron tanto con el parámetro $m$ libre (cuadro 5) como constante (cuadro 6). Con ello es posible evaluar a través de los estadísticos tradicionales de la regresión lineal la calidad de los ajustes. En el cuadro 6 es posible apreciar que los coeficientes de determinación superan el 98\% de explicación, mientras que los errores estándares son inferiores a $0,05 \mathrm{~cm} / a n ̃ o$.

Los parámetros de la función de rendimiento [2], obtenidos por la transformación de la función [4], se presentan en el cuadro 7, en el cual también se observan los parámetros de vida que se obtienen a partir de los ajustes. Hay coincidencia con los resultados de los modelos anteriores, respecto a la más temprana culminación (TPI) del incremento diamétrico de los árboles suprimidos, como así también del dap que poseen dichos árboles en tal momento (DPI). Respecto de los tiempos de vida (TV), con ambos grupos de datos se obtuvieron estimaciones menores a los 300 años, coincidiendo los respectivos a los estratos superior e intermedio, pero sensiblemente diferentes los del estrato suprimido.

Los resultados dados por los ajustes de las funciones para las series de crecimiento revelan la dinámica promedio de los árboles en el área estudiada y para períodos de hasta 140 años, aproximadamente. Las edades límites dadas por los datos disponibles han restringido los estudios a las mismas. 
CUADRO 5

Parámetros de la función de rendimiento [2] para series de crecimientos de cada estrato de $N$. obliqua y los parámetros biológicos relacionados.

Parameters of function [2] from the average real growth series of the $N$. obliqua social stratum and related biological parameters.

\begin{tabular}{|l|c|c|c|c|c|c|c|c|c|}
\hline Estrato & $m$ & $D$ & $k$ & $\begin{array}{c}\text { MCA } \\
{[\mathrm{cm} / \mathrm{año}]}\end{array}$ & $\begin{array}{c}\text { TV } \\
{[\mathrm{años}]}\end{array}$ & $\begin{array}{c}\text { DPI } \\
{[\mathrm{cm}]}\end{array}$ & $\begin{array}{c}\text { CRPI } \\
{[\%]}\end{array}$ & $\begin{array}{c}\text { Máx.ICA } \\
{[\mathrm{cm} / \mathrm{año}]}\end{array}$ & $\begin{array}{c}\text { TPI } \\
{[\mathrm{años}]}\end{array}$ \\
\hline Superior & 0,0962495 & 74,0 & 0,00991018 & 0,33 & 221 & 5,6 & 10 & 0,571 & 10 \\
Intermedio & 0,0726778 & 62,0 & 0,008022765 & 0,23 & 267 & 3,7 & 11 & 0,405 & 9 \\
Suprimido & 0,0429453 & 29,0 & 0,01589781 & 0,22 & 131 & 1,1 & 37 & 0,402 & 3 \\
\hline
\end{tabular}

\section{CUADRO 6}

Parámetros y estadísticos obtenidos por regresión no lineal de la función [4], a partir de los promedios de las series de crecimientos y con el parámetro m libre.

Parameters and statistics obtained from a non-linear fitting of equation [4] from the average real growth series and with parameter $\mathrm{m}$ being free.

\begin{tabular}{|l|c|c|c|}
\hline Estrato & Parámetro $a$ & Parámetro $D$ & Parámetro $m$ \\
\hline Superior & 0,536231 & 74,0 & 0,0962495 \\
Intermedio & 0,397391 & 62,0 & 0,0726778 \\
& 0,0429453 \\
Suprimido & 0,416865 & 29,0 & \\
\hline Estrato & $E A M[\mathrm{~cm}]$ & $E E E[\mathrm{~cm}]$ & $S C R\left[\mathrm{~cm}^{2}\right]$ \\
\hline Superior & 0,0279 & 0,0482 & 0,0209 \\
Intermedio & 0,0299 & 0,0396 & 0,0094 \\
Suprimido & 0,0194 & 0,0325 & 0,0032 \\
\hline
\end{tabular}

Las significancias biológicas de estas funciones son diferentes a las obtenidas con datos independientes, pues justamente la interdependencia de los datos de cada serie permite describir la dinámica para los valores promedios del conjunto de árboles estudiados, que en la actualidad pertenecen al mismo estrato social. Y respecto a este punto hay una serie de consideraciones importantes. Primero, de la misma manera que con los datos independientes, el hecho de que un árbol pertenezca hoy a un determinado estrato de competencia, no significa que sea al que haya pertenecido a lo largo de su vida, la importante diferencia es que ahora se podría llegar a inferir cuáles fueron sus estados de competencia anteriores. Segundo, si las

\section{CUADRO 7}

Parámetros y estadísticos obtenidos por regresión lineal múltiple de la función [4], a partir de los promedios de las series de crecimientos, con el parámetro m fijo para cada estrato social de $N$. obliqua.

Parameters and statistics obtained from a multiple linear fitting of equation [4] of the average real growth series, with parameter $\mathrm{m}$ as a constant for each $N$. obliqua social stratum.

\begin{tabular}{|l|c|c|c|}
\hline Estrato & Parámetro $a$ & Parámetro $D$ & Parámetro $m$ \\
\hline Superior & 0,524322 & 76,8 & 0,0962495 \\
Intermedio & 0,376517 & 71,0 & 0,0726778 \\
Suprimido & 0,405955 & 31,0 & 0,0429453 \\
& & & \\
\hline Estrato & $R^{2}[\%]$ & EEE $[\mathrm{cm}]$ & $S C R\left[\mathrm{~cm}^{2}\right]$ \\
\hline Superior & 98,8 & 0,0472 & 0,0200 \\
Intermedio & 98,9 & 0,0357 & 0,0076 \\
Suprimido & 99,0 & 0,0303 & 0,0028 \\
\hline
\end{tabular}

series pertenecen a árboles de muy diferentes tamaños y se las confronta en un mismo gráfico, se podría estar relacionando para un mismo momento en la vida de ambos crecimientos de árboles de diferentes estratos. Por ello podría darse el caso de estar promediando, por ejemplo, el crecimiento actual de una planta oprimida de $25 \mathrm{~cm}$, con el de otra que actualmente es oprimida con un dap de $40 \mathrm{~cm}$, pero que cuando tenía $25 \mathrm{~cm}$ podría no haberlo estado.

Esta última consideración limita el estudio a los árboles de más edad, es decir, si se espera que los árboles en estratos intermedios alcancen diámetros máximos de $60 \mathrm{~cm}$, los análisis de las dinámicas deben basarse en árboles de al menos 

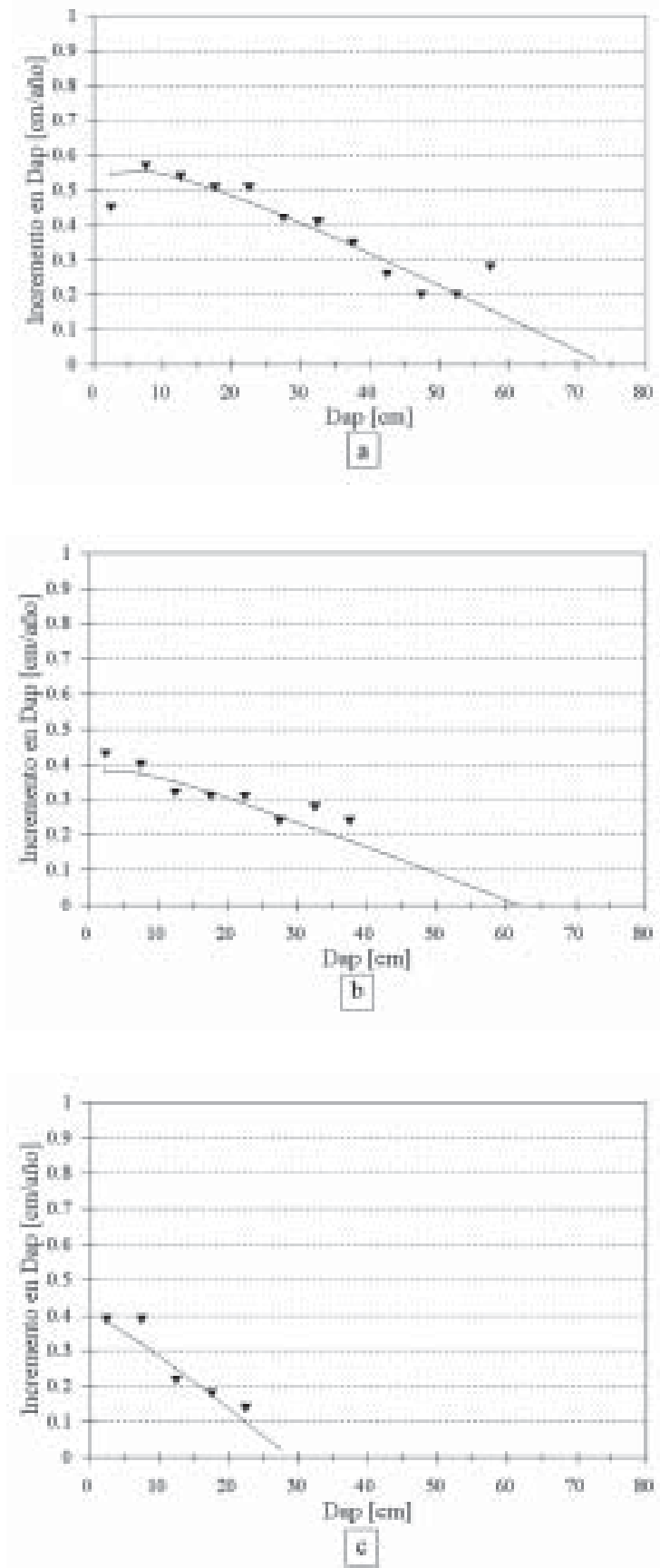

Figura 2: Función [4] ajustada a los valores de incrementos diamétricos anuales promedios a partir de las series de crecimientos, para diferentes estratos sociales: a) estrato superior; b) estrato intermedio y c) estrato suprimido.

Parameters and statistics obtained from a multiple linear fitting of equation [4] of the average real growth series, with parameter $\mathrm{m}$ as a constant for each $N$. obliqua social stratum. diámetros de 45-50 cm. Las muestras utilizadas presentan mayoritariamente edades entre 80 y 120 años para $N$. obliqua; considerando que pueden llegar a edades entre 250-300 años, las posibilidades de extrapolación para inferir la dinámica por encima de estas edades dependerá de lo adecuado de los valores de los parámetros de las funciones.

Las similares culminaciones del crecimiento del estrato suprimido estimadas para ambos grupos de datos señalan una historia equivalente ${ }^{1}$, lo que estaría indicando que la especie es muy sensible a la competencia por los recursos del medio. Pero se ha de notar cierta duda respecto a los niveles de incrementos y las edades de culminación de ambos grupos. Las series de crecimientos culminan a los 3 años con un incremento de $0,40 \mathrm{~cm} / \mathrm{año}$, mientras que los datos independientes lo hacen a los 6 años con 0,23 cm/año. Antes de ensayar cualquier explicación se le atribuye la inconsistencia a la falta de datos independientes en la clase de diámetro $<5 \mathrm{~cm}$, clase clave para interpretar este estrato social. Estos constituyen los resultados más delicados ya que así como están, $N$. obliqua disminuiría su crecimiento sensiblemente al menor síntoma de supresión, pero también estarían indicando que es capaz de soportar en esta condición hasta alcanzar diámetros de $25-30 \mathrm{~cm}$ que, bajo estos niveles de crecimiento, se alcanzarían entre los 100 y 130 años. Es decir, el fenómeno de autorraleo en masas regulares de $N$. obliqua, ocurriría durante el período inicial de su vida, pues los árboles suprimidos no tendrían posibilidades de alcanzar estas edades.

Consideraciones generales. Se encontraron diferencias entre los valores absolutos promedios de los incrementos (MCA) (cuadros 4 y 7), pero éstos pueden ser tomados como una referencia, pues dicho promedio considera todo el tiempo de vida (TV) de cada función respectiva, que, como se ha visto, son variables según el grupo. O lo que es lo mismo, promedia los incrementos de todas las clases diamétricas y por las razones expuestas se pueden estar promediando incrementos de clases no naturales, como es el caso cuando se utilizan funciones con diámetros asintóticos muy altos.

\footnotetext{
Recordar que la función ajustada con datos independientes representa a un árbol que crece en el mismo estrato social a lo largo de su vida.
} 
Si se analizan los valores máximos de incrementos corrientes anuales, dados en los cuadros 4 y 7 , se pueden realizar algunas inferencias. En primer lugar, bajo la hipótesis de que los dominantes constituyen un estrato estable por largos períodos, hasta incluso toda la vida del individuo, podría esperarse entonces que no aparezcan diferencias entre los valores máximos del ICA; sin embargo, se encontraron diferencias entre dichos valores máximos y ello puede atribuirse a una variación lógica natural del bosque estudiado.

La diferencia entre los incrementos máximos del estrato intermedio se considera también atribuible a variaciones naturales, por lo que al momento de producirse la culminación (en ambos grupos ocurrió al mismo tiempo), el árbol ya se encuentra sociológicamente definido en un estrato de crecimiento o competencia inferior.

El estrato suprimido termina de caracterizar la dinámica de la competencia; los valores máximos encontrados en las series de crecimiento son levemente inferiores a los máximos del estrato intermedio, lo que se interpreta como que la tendencia del desarrollo futuro, en un estrato intermedio (recibe radiación solar directa) o suprimido (no recibe radiación solar directa), recién se está produciendo en los momentos de ocurrencia de las culminaciones de cada especie.

Los estratos superior e intermedio presentan similitudes entre los dos grupos de datos en las culminaciones de los crecimientos corrientes anuales, cercanas a los 10 años y, como se expuso precedentemente, con niveles de incrementos también similares en cada estrato. El hecho de que el estrato intermedio esté recibiendo luz directa puede ser suficiente para mantener un patrón de crecimiento natural y similar al de los árboles dominantes, pero a un ritmo menor.

De acuerdo a los resultados, la hipótesis de que el estrato intermedio es el menos estable en el tiempo queda en duda, al menos para la primera mitad del ciclo de vida estimado. La similitud para los primeros 120 años de vida de los patrones de crecimiento de ambos grupos de datos confirma que una vez que el individuo alcanzó el canopeo superior como estrato intermedio es capaz de mantenerse en esa condición hasta la madurez o se produzca la liberación. De acuerdo a estudios realizados en estos bosques (Chauchard et al. 1996), el estrato intermedio representa aproximadamente el $10 \%$ de la población madura, ahora se puede suponer que estos árboles, en bosques regulares, podrían haber alcanzado esta condición en su juventud y mantenerse así hasta la madurez. La pregunta que queda por responder es ¿hasta qué edad o tamaño un individuo de $N$. obliqua es capaz de responder a una liberación?

El estrato suprimido es el que presenta mayores diferencias entre los dos grupos y ello puede estar marcado por la dinámica natural de competencia, proceso que estaría siendo descrito por la curva ajustada con las series de crecimiento.

Para árboles que crecen en grupos regulares de edades, la diferenciación de estratos es un proceso de competencia que se define principalmente en la etapa juvenil, salvo los renovales que nacieron ya bajo la competencia de un dosel superior; un individuo que alcanza cierta edad como suprimido puede haber sido como resultado de haber sucumbido en la lucha por los recursos del micrositio que ocupan. La función dada por los datos independientes se podría asimilar para aquellos casos en que el renoval pasa su vida hasta su muerte bajo una fuerte opresión de sus pares, pero si así fuera, difícilmente tendría la evolución dada por esta función, ya que la muerte sobrevendría antes de alcanzar los diámetros mayores de este estrato.

Comparando las culminaciones de los incrementos corrientes en diámetro se puede inferir que las diferenciaciones de los estratos sociales son tempranas. Hasta los 5 años se estarían diferenciando los estratos dominantes y suprimidos y entre los 5 y 15 años, aproximadamente, se diferenciaría el estrato intermedio de los dominantes.

También se puede apreciar que los incrementos de los árboles dominantes e intermedios tienden a igualarse a partir de cierta edad. Tal proceso sólo podría explicarse en el caso de que los árboles intermedios vayan paulatinamente ocupando posiciones dominantes, pero tal resultado se considera que se ha producido por extrapolar algunas de las funciones ajustadas. Tal inconsistencia sólo se puede remediar con series de crecimientos de edades mayores que las disponibles para esta investigación.

A la luz de los resultados se vuelve a poner en duda la hipótesis de rendimiento final común para un sitio dado (Piennar 1969, Vázquez 1988) cuando se estudian los crecimientos a nivel individual. Ratificando lo encontrado en $N$. nervosa (Chauchard 1991) y en N. dombeyi (Chauchard et al. 2001), se 
ha demostrado que los individuos que crecen en un mismo sitio pero bajo diferentes presiones competitivas no alcanzarán las mismas dimensiones finales.

En la dinámica natural del bosque de Nothofagus puede ocurrir que un individuo pase por etapas sucesivas de opresión y liberación y por el fenómeno de competencia se va cumpliendo la selección natural, que principalmente actúa sobre los individuos que desarrollan en inferioridad de condiciones. Estos individuos, que poseen el potencial suficiente, pero desarrollan en condiciones desventajosas, nunca alcanzarán las dimensiones de aquellos individuos que aprovechan al máximo los elementos del sitio (Chauchard 1991).

\section{CONCLUSIONES}

- El modelo de Bertalanffy-Richards estudiado posee una gran flexibilidad y se ha adaptado satisfactoriamente a los distintos grupos de datos con los que se ha trabajado. Pero dicha flexibilidad puede ser riesgosa, dado que permitiría obtener ajustes de validez estadística pero de poca consistencia biológica, por lo que se deben extremar las precauciones al momento de analizar los modelos ajustados.

- El conocimiento de los factores biológicos asociados al fenómeno de crecimiento estudiado y de las características del modelo han permitido conseguir buenos ajustes de los modelos, balanceando entre los resultados aceptables estadística y biológicamente. Con dichos modelos se abre una puerta para el desarrollo de regímenes silvícolas de los rodales de $N$. obliqua.

- Se rechaza la hipótesis de asíntota común cuando se modela el crecimiento de árboles individuales de diferentes estratos sociales. El crecimiento de la especie estudiada está positivamente correlacionado con la posición sociológica que ocupen en el canopeo y por ende con el volumen de la copa. El tamaño final del árbol y el momento de culminación de su incremento corriente anual está correlacionado con el estrato de competencia que vaya ocupando a lo largo de su vida; a mejor estrato de competencia mayor tamaño final se alcanza. En particular, a la culminación del incremento corriente anual éste es anterior en árboles suprimidos, respecto de los dominantes, no así la de los intermedios que presentan una época de culminación semejante a la de los dominantes.

- $N$. obliqua ha mostrado que puede alcanzar buenos crecimientos máximos anuales, en condiciones naturales promedio de la zona estudiada, pudiendo superar los 0,6 cm/año en etapas juveniles.

- Analizando comparativamente los resultados de los anteriores estudios (Chauchard 1991, Chauchard et al. 2001), N. obliqua ha presentado una culminación más temprana de sus crecimientos corrientes anuales respecto de $N$. dombeyi. Los momentos de culminaciones ocurren en $N$. obliqua hasta los 10 años, $N$. dombeyi hasta los 30 años. Sumando los resultados anteriores sobre $N$. nervosa, el orden de tolerancia a la sombra, de mayor a menor es: $N$. nervosa, $N$. dombeyi y N. obliqua.

- La especie estudiada define su posición social y su tendencia al desarrollo en la fase juvenil, y puede mantenerse creciendo en los estratos dominante e intermedio hasta la fase de madurez. En masas regulares la diferenciación del estrato suprimido se produce hasta los cinco años (edad al dap) y hasta los 15 años se produce la diferenciación de los dominantes del intermedio. Esta diferenciación temprana señala que $N$. obliqua es sensible a la competencia, produciéndose su mortandad y autorraleo antes de los 130 años de edad al dap.

\section{BIBLIOGRAFIA}

CHAUCHARD, L. 1991، "Modelos de crecimiento individual del Raulí (Nothofagus alpina (Poepp. et Endl.) Oerst)", Actas VI Jornadas Técnicas: Inventario, Modelos de Producción y Crecimiento Forestales. Eldorado, Misiones: 246-276.

CHAUCHARD, L., M. GONZALEZ PEÑALBA, L. MARESCA, A. RABINO, R. SBRANCIA. 1996. Informe Final Proyecto Dinámica y manejo de bosque de Nothofagus. San Martín de los Andes. UNC-INTA-APN. Inédito. 93 p.

CHAUCHARD, L., R. SBRANCIA, M. GONZALEZ PEÑALBA, L. MARESCA, A. RABINO. 1999. "Aplicación de leyes fundamentales de la densidad a bosques de Nothofagus: I. Regla de los $-3 / 2$ o ley del autorraleo", Bosque 20(2): 79-94.

CHAUCHARD, L., R. SBRANCIA, A. RABINO, M GONZALEZ PEÑALBA, L. MARESCA. 2001. "Modelos de crecimiento diamétrico para Nothofagus dombeyi", Bosque 22(2): 53-68.

DONOSO, C. 1993. Bosques templados de Chile y Argentina. Variación, estructura y dinámica. Ecología forestal. Santiago de Chile, $1^{\text {ra }}$. Ed., Universitaria S.A. 484 p. 
BOSQUE 24(3): 3-16, 2003

Modelos de crecimiento diamétrico para Nothofagus obliqua

DRAPER, N., H. SMITH. 1981. Applied regression analysis. New York, $2^{\text {nd }}$ ed., John Wiley \& Sons. 673 p.

KLEPAC, D. 1983. Crecimiento e incremento de árboles y masas forestales. Chapingo, Universidad Autónoma de Chapingo. $365 \mathrm{p}$.

OSUMI, S. 1983. Applicabilité de la fonction de Richards a l'analyse de croissance de l'arbre. Les Colloques de l'INRA (17): 77-86

PERI, P., G. MARTINEZ. 1997. “Crecimiento diamétrico de Nothofagus pumilio para dos condiciones de copa en un sitio de calidad media de Santa Cruz (Argentina)", Revista de Investigación Agraria: Sistemas y Recursos Forestales 5 (2).

PIENAAR, L. 1969. "Towards the development of forest management tables", Forestry in South Africa (10): 15-19. RICHARDS, F. 1959. "A flexible growth function for empirical use”, Jour. of Exp. Bot. 10 (29): 290-300.
SBRANCIA, R., A., RABINO, S., GOICOECHEA. 1998. Plan de ordenación forestal: cantones Trompul, Abanico y Pil Pil. San Martín de los Andes, Universidad Nacional del Comahue. Inédito, $51 \mathrm{p}$.

SPURR, S., B. BARNES. 1980. Ecología Forestal. México,

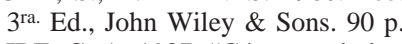

URIBE, G. A. 1987. "Cómputo de la regresión lineal múltiple y de la regresión no lineal", Crónica Forestal y del Medio Ambiente 3 (1 y 2): 124-179.

VANCLAY, J. 1994. "Synthesis-growth models for tropical forests: a synthesis of models and methods", For. Sci. 41 (1): 7-42.

VAZQUEZ, V. 1988. "Crecimiento de un bosque de Guadal explotado en el litoral pacífico colombiano: estudio del crecimiento diamétrico (I)", Crónica Forestal y del Medio Ambiente, junio: $35-62 \mathrm{p}$.

ZEIDE, B. 1989. "Accuracy of equations describing diameter growth", Can. Jour. of For. Res. 19: 1283-1286. 